\title{
Design of Terahertz Waveband Antenna Based on Fractal Photonic Crystal Structure
}

\author{
Bin Lin ${ }^{1, \text { a }}$, Chang Lin ${ }^{1}$, Yunhai Mao ${ }^{1}$, Zhihang Chen ${ }^{1}$, Peitao Zhang ${ }^{1}$, Yuankun \\ $\mathrm{Cai}^{1}$, Guangya Ye ${ }^{1}$, Yu Zhang ${ }^{1}$ \\ ${ }^{1}$ Xiamen University Tan Kah Kee College, Fujian Zhangzhou 363105, China \\ aemail: linbin@xujc.com
}

Keywords: Terahertz Waveband Antenna; Cantor Fractal Structure; Photonic Crystal Structure; Wave-Transmitting Ceramic Substrate.

Abstract. In terms of the performance requirements of Terahertz waveband antenna on Terahertz devices, the present paper originally combines Cantor fractal structure, photonic crystal structure and Terahertz waveband wave-transmitting ceramic substrate, designing a Terahertz waveband antenna based on fractal photonic crystal structure, making antenna samples to run test, discussing the effect on the performance of the antenna with changes of parameters of dielectric substrates. According to the test results, this antenna's working central frequency is $1 \mathrm{THz}$, the minimum of its return loss value is less than $-15 \mathrm{~dB}$, absolute working bandwidth and relative working bandwidth are greater than $0.1 \mathrm{THz}$ and $10 \%$, respectively. With the characteristics of small size, high radiation performance, wide working bandwidth and easy integration, this antenna has a wide horizon of application in the Terahertz waveband field.

\section{Introduction}

Terahertz wave is a type of electromagnetic wave whose frequency is in the range of 0.1 to $10 \mathrm{THz}$. Its electromagnetic radiation area is located between microwaves and infrared wave band. For quite long time, this area was the gap of electromagnetic wave spectrum due to the lack of effective ways to generate and test Terahertz waves. The both sides of Terahertz wave band, infrared- related and microwaves-related technologies had become quite mature. However, the development of Terahertz technology is relatively slow. Since 1980s, the development of free electron laser and ultrafast technology provides the generation of Terahertz with stable and reliable excitation source, which makes the mechanism, detection and applied technology research of Terahertz flourish. The Terahertz spectrum of matters has rich physical and chemical information [1-2], which lets Terahertz electromagnetic wave have the great scientific research value and board perspectives in the fields of object imaging, environment monitoring, medical diagnosis, radio-astronomer, broadband mobile communication and military radar [3-4].

The application of Terahertz wave could not work without the Terahertz transmit and receive devices. Excellent performance of Terahertz waveband antenna has great significance on the application of Terahertz. wave The requirements of current Terahertz devices for the Terahertz waveband antenna are: relatively small size, could be put into Terahertz devices with millimeter orders of magnitude, working central frequency is around $1 \mathrm{THz}$, minimum of return loss value less than $-15 \mathrm{~dB}$, absolute working bandwidth and relative working bandwidth greater than $0.1 \mathrm{THz}$ and $10 \%$, respectively.

\section{Introduction of Photonic Crystal Structure}

The definition of photonic crystal was promoted by Yablonovitch and John in 1987 when they did the research about how to control spontaneous radiation and about features of photon localization, respectively. It is basically a new type of photonic microstructure materials, which has photonic band with the spatial periodic changes of refractive index. Its periodical variation is optical-wavelength orders of magnitude and it has gained success in the applications of the microwave-waveband-devices. In terms of different arrangements of dielectric that composes the 
photonic crystal, there are three general types of structures of photonic crystals: one-dimensional, two-dimensional and three-dimensional.

Due to the reasons related to manufacturing process and so on, photonic crystal hasn't been widely used at present. The size of lattice that produces photonic crystal is equivalent as the wavelength of light. The smaller size of photonic crystal is, the more difficult manufacturing will be. For instance, the size of photonic crystal lattice that lies in the waveband of visible light(380-780nm) and near infrared(780-3000nm) is relatively small, but that in microwave band is centimeter orders of magnitude, to make it easier to manufacture. However, its comparatively big size limits its realistic applications. In contrast, Terahertz wave locates between these two wavebands to make manufacturing process and produce Terahertz waves in labs experiments is not hard and its physical size is not so much big. All these features provide feasibility for the applications of Terahertz waveband devices based on photonic crystal structure and Terahertz systems.

Photonic crystal has two basic characteristics, for one is photonic bandgap, the photons fall into bandgap could not propagate. The electromagnetic waves whose frequency lies in photonic bandgap could not propagated through photonic crystal. Based on this feature, researchers have developed many kinds of microwave devices and optical wave devices, including high efficiency reflector, high R-factor Micro-cavity, wideband band-stop filter, ultra narrow band selective filter, optical switch and photonic crystal fiber [5-6]. For our research on Terahertz waveband, photonic crystal also has its applicable potentials. Another feature of photonic crystal is photonic localization. Introducing defects to some degree into the photonic crystal, the photons whose frequency tallies with that of defect frequency will propagate through localization, to make it possible for the direction propagation of electromagnetic waves.

\section{Introduction of Cantor Fractal Structure}

For devices operated in Terahertz waveband, wideband operation capability will also be one of the key requirements. and fractal techniques are not doubt one of the efficient ways to achieve that. The research about the mutual interaction between waves and fractal structures promoted the development of fractal electrodynamics in 1980s to make us could efficiently design devices with the features of miniaturization and wideband operation [7-8].

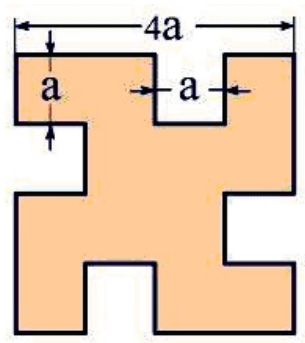

(a) 1st-order Cantor fractal structure

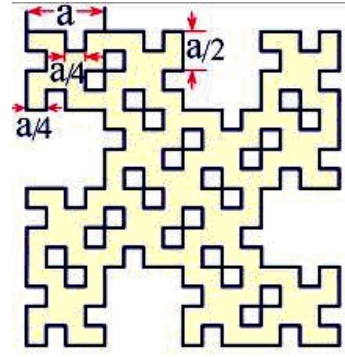

(b) 2nd-order Cantor fractal structure

Fig.1. The scheme of Cantor fractal structure

The fractal structure applied in this antenna is Cantor fractal structure. As it is shown in Figure 1, the initial element of Cantor fractal structure is a square. After bisecting it into four rows four columns to come out with 16 smaller squares and removing the third square of the first row, the first square of the second row, the forth square of the third row and the second square of the forth row to remain 12 small squares left, it becomes the first order Cantor fractal structure. After bisecting each small square of first order Cantor fractal structure into 16 smaller squares of 4 rows 4 columns and removing the third square of the first row, the first square of the second row, the forth square of the third row and the second square of the forth row, it becomes the second order Cantor fractal structure. Following this pattern, we could generate higher orders of Cantor fractal structure. 


\section{Design of Terahertz Waveband Antenna Structure}

We use low-loss wave-transmitting ceramic materials and fractal mental chips as the basic material of Terahertz waveband fractal photonic crystal structure. We have independent intellectual property rights about low-loss ceramic materials, which overcome the problems of conventional high permittivity materials that would result in large loss for high frequency signals and have successfully applied it into Terahertz waveband devices, obtaining a license of invention patent in China. This type of ceramic materials takes ceramic powders, dispersant and Carrageenan as row materials to produce this with multiple manufacturing processes. According to the test result of microwave test center in the University of Electronic Science and Technology of China, the permittivity of it is between 10 and 100 and dissipation factor is smaller than 0.002 to make it particularly appropriate to be substrate materials for Terahertz waveband devices.

Photonic crystal structure is composed of a periodic arrangement of one kind of dielectric in another kind of dielectric. We use fractal mental chips as the micro radiation structure of antennas, applying magnetron sputtering technics to let fractal mental chips distribute periodically in ceramic materials, composing fractal photonic crystal structure. With the appropriate design of feed, this type of fractal photonic crystal structure composed by fractal mental chips and ceramic materials could be fractal photonic crystal antennas to generate radiation in the Terahertz waveband. The structure of Terahertz waveband antenna based on fractal photonic crystal structure is shown in Figure 2 and Figure 3.

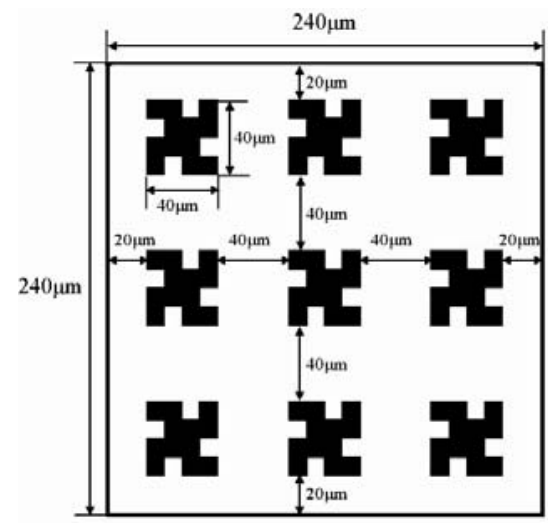

Fig.2. The scheme of antenna's fractal photonic crystal radiation chips

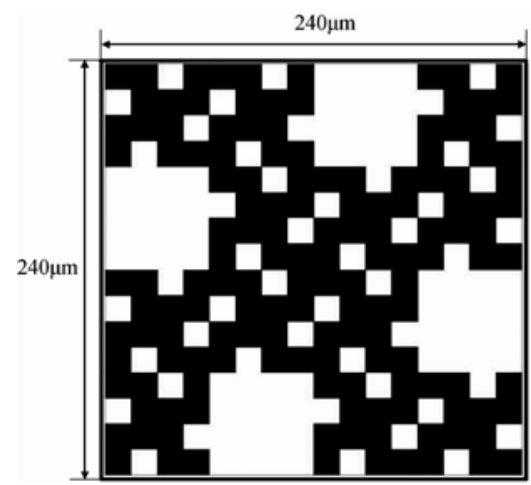

Fig.3. The scheme of antenna's grounding structure

The antenna is composed of grounding structure and fractal photonic crystal radiation chips. They are located in two sides of substrate board respectively. Substrate board is the Terahertz waveband wave-transmitting ceramic material, its permittivity is 14 , the figure is rectangle and the size is $240 \mu \mathrm{m} \times 240 \mu \mathrm{m}$ with the thickness at $10 \mu \mathrm{m}$.

The fractal photonic crystal radiation chips of antenna is composed of 3 rows 3 columns; 9 fractal smaller antennas on ceramic substrate board by the periodic distribution of 3 rows 3 columns array. The size of each fractal mental antenna is $40 \mu \mathrm{m} \times 40 \mu \mathrm{m}$ and each fractal mental antenna uses first order Cantor fractal structure. In these 9 fractal mental antennas of 3 rows 3 columns, the first row is $20 \mu \mathrm{m}$ away from the top of the substrate board and the distance of the first row to the second row and the second row to the third row are all $40 \mu \mathrm{m}$. The third row is $20 \mu \mathrm{m}$ away from the bottom of the substrate board. The first column is $20 \mu \mathrm{m}$ away from the left of the substrate board and the distance of the first column to the second column and the second column to the third column are all $40 \mu \mathrm{m}$. The third column is $20 \mu \mathrm{m}$ away from the right of the substrate board. The grounding structure of antennas is the second order Cantor fractal structure with the same size as the substrate board.

We unite the photonic crystal technics and fractal technics to combine their respective advantages, efficiently improving the performance of the Terahertz waveband antenna. Mental radiation chips are distributed periodically in the ceramic materials to produce the photonic crystal structure, introducing fractal evolution patterns in each single periodic unit to produce fractal photonic crystal structure. 
The antenna based on this structure has both characteristics of high radiation and board working bandwidth. The grounding structure is designed as the second order Cantor fractal structure, which helps even electricity distribution on antennas to enhance its capability of working bandwidth.

\section{The manufacture and test of the Terahertz waveband antenna based on fractal photonic crystal structure}

As is stated above, we use magnetron sputtering technics to produce samples of Terahertz waveband antenna based on fractal photonic crystal structure and we run the test for the performance of its radiation characteristics and the result is shown in Figure 4.

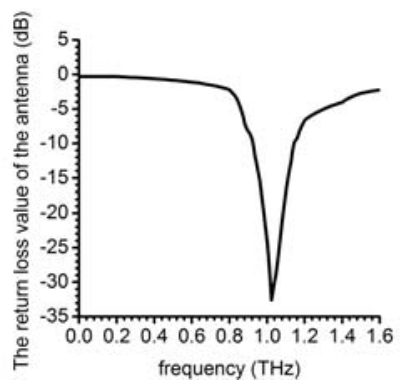

Fig.4. The antenna's radiation characteristics test results

From Figure 4 we can observe that the working center frequency of this antenna is $1.02 \mathrm{THz}$; the minimum of its return loss value is $-32.58 \mathrm{~dB}$; antenna's working frequency range is between 0.926 and $1.143 \mathrm{THz}$; antenna's absolute working bandwidth and relative working bandwidth are 0.217 $\mathrm{THz}$ and $21.27 \%$, respectively. The test result indicates that this antenna could fulfill the performance requirements of Terahertz waveband antennas for current Terahertz devices.

\section{The effect on the performance of the antenna with changes of parameters of dielectric substrates}

In our design description, relative dielectric constant of wave-transmitting ceramic substrate is 14 . In real manufacture, however, because of differences of different ceramic powders, dispersant and manufacturing processes, the relative dielectric constant may have kind of bias, so it is necessary to discuss the effect on the performance of the antenna with changes of the relative dielectric constant of dielectric substrates. Under the circumstance of constant thickness of substrate at $10 \mu \mathrm{m}$, we run a series of computing simulations by changing the relative dielectric constant of wave-transmitting ceramic substrates and the result is shown in Figure 5.

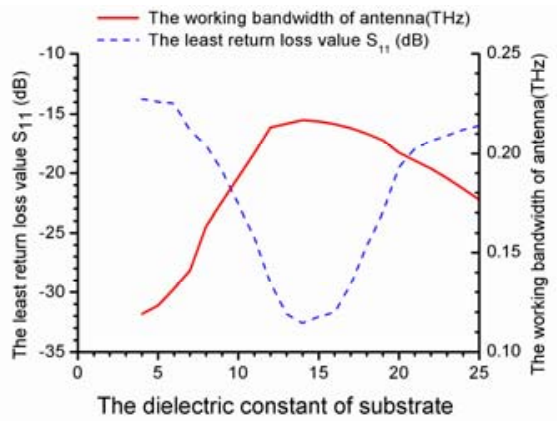

Fig.5. The effect on the performance of the antenna with changes of parameters of dielectric substrates

Figure 5 shows that with the decrease of the relative dielectric constant, the minimum value of $\mathrm{S}_{11}$ of antenna declines and the working bandwidth is getting boarder. This is because when the relative dielectric constant decreases, the restraint from dielectric to field reduces to make it easy to radiate and stored energy reduces with the decrease of the relative dielectric constant as well, resulting in quality factor of antennas decreases and antennas apply more energy for radiation to board working 
bandwidth, which means advisably decreasing the relative dielectric constant of substrate board could improve the performance of antenna.

But when the relative dielectric constant decreases to some degree $\left(\varepsilon_{r}<14\right)$, it will cause that minimum value of $\mathrm{S}_{11}$ of antenna increases and working bandwidth would narrow gradually, which means in the case of sharp changes of the relative dielectric constant, the matching of antenna will wreck and the radiation and bandwidth performance of antenna will be weak as well.

From what has been discussed above, beyond the volume requirement of Terahertz devices and the antenna matching capability, appropriately reducing the relative dielectric constant of the substrate board could enhance the performance of return loss and bandwidth for antenna.

\section{Conclusions}

In terms of the performance requirements of Terahertz waveband antenna on Terahertz devices, we use low-loss wave-transmitting ceramic materials as the substrate board, combing Cantor fractal structure and photonic crystal structure to design Terahertz waveband antenna based on fractal photonic crystal structure and to run tests for antenna samples, discussing the effect on the performance of the antenna with changes of parameters of dielectric substrates. The antenna size is small at $240 \mu \mathrm{m} \times 240 \mu \mathrm{m} \times 10 \mu \mathrm{m}$ and it could be put into Terahertz devices with millimeter and submillimeter orders of magnitude. Antenna's working center frequency is $1.02 \mathrm{THz}$; the minimum of its return loss value is $-32.58 \mathrm{~dB}$; antenna's absolute working bandwidth is $0.217 \mathrm{THz}$. This antenna could fulfill the performance requirements of Terahertz waveband antennas for current Terahertz devices with characteristics of high performance of radiation, board working bandwidth, potential performance redundancy and capability of working in harsh environments. With features of small size, high radiation performance, wide bandwidth and easy integration, this antenna has a wide horizon of application in the Terahertz waveband field.

\section{References}

[1] Yang Li, Xie Yanhua, Ding Yuanming and Song Lin. ICIC Express Letters Vol. 2 (2013), p. 491-496.

[2] Siegel P H. IEEE Transactions on Microwave Theory and Techniques Vol. 3 (2002), p. 910-928.

[3] Ta Jin-Xing, Han Yu and Lan Cheng. Journal of Magnetism and Magnetic Materials Vol. 399 (2016), p. 245-251.

[4] Faraji Mahboobeh, Moravvej-Farshi Mohammad Kazem and Yousefi Leila. Optics Communications Vol. 355 (2015), p. 352-355.

[5] Fink Y, Winn J N and Fan S. Science Vol. 282 (1998), p. 1679-1682.

[6] Kopp V I, Fan B and Vithana H K M. Opt. Lett Vol. 23(1998), p. 1707-1709.

[7] Tripathi Shrivishal, Mohan Akhilesh and Yadav Sandeep. Microwave and Optical Technology Letters Vol. 11 (2014), p. 2469-2473.

[8] Rani Shweta and Singh A.P. International Journal of Applied Electromagnetics and Mechanics Vol. 2 (2013), p. 259-267. 